\title{
Chyloperitoneum as the Initial Manifestation of Gastrointestinal Neoplasia
}

\author{
Ivan David Lozada-Martínez ${ }^{1, * \mathbb{D}}$, Daniela Torres-Llinás ${ }^{1}$, Paola Zuluaga-Ramírez ${ }^{2}$, \\ Victor Hugo Mendoza-Brochero ${ }^{3}$, María Bolaño-Romero ${ }^{1}$ and Ana María Ríos-Giraldo ${ }^{3,4}$
}

1 Medical and Surgical Research Center, Faculty of Medicine, University of Cartagena, Cartagena 130014, Colombia; dtorres1@unicartagena.edu.co (D.T.-L.); mariapazboro2016@gmail.com (M.B.-R.)

2 Surgical Department, Faculty of Medicine, University of Cartagena, Cartagena 130014, Colombia; zuluagapaola1909@gmail.com

3 Surgical Department, San Jorge University Hospital, Pereira 660009, Colombia; Dr.victormendoza@gmail.com (V.H.M.-B.); idlozadam@gmail.com (A.M.R.-G.)

4 Medical Department, Autonomous University Foundation of the Americas, Pereira 660009, Colombia

* Correspondence: ivandavidloma@gmail.com

check for

updates

Citation: Lozada-Martínez, I.D.; Torres-Llinás, D.; Zuluaga-Ramírez, P.; Mendoza-Brochero, V.H.;

Bolaño-Romero, M.; Ríos-Giraldo, A.M. Chyloperitoneum as the Initial Manifestation of Gastrointestinal

Neoplasia. Surgeries 2021, 2, 167-173. https://doi.org/10.3390/

surgeries2020017

Academic Editor: Cornelis F. M. Sier

Received: 24 March 2021

Accepted: 21 April 2021

Published: 22 April 2021

Publisher's Note: MDPI stays neutral with regard to jurisdictional claims in published maps and institutional affiliations.

Copyright: (c) 2021 by the authors. Licensee MDPI, Basel, Switzerland. This article is an open access article distributed under the terms and conditions of the Creative Commons Attribution (CC BY) license (https:/ / creativecommons.org/licenses/by/ $4.0 /)$.

\begin{abstract}
Chyloperitoneum is defined as the presence of lymph within the peritoneal cavity, resulting from obstruction or injury of lymph ducts, mostly at the level of the gastrointestinal tract. This can occur in the context of congenital diseases, traumas, infections, neoplasms, hepatic disease, heart disease, and postoperative complications. The most common symptoms described are abdominal distention and mild pain in a course of weeks to months, with dyspnea, peritonitis, and in a few cases weight gain is observed due to the high intra-abdominal pressure. We present a case of a 56-year-old male with no significant personal history, who has a clinical picture of approximately three months of evolution, consisting in sensation of an abdominal mass predominantly in the left hemiabdomen, associated with progressive abdominal distension, changes in intestinal habit, lower limb edema, dyspepsia, occasional postprandial emesis, and unintentional weight loss of $20 \mathrm{~kg}$. In non-traumatic conditions, the most frequent cause of chylous ascites is a malignancy disease followed by cirrhosis and mycobacterial infections. Taking into consideration that adenocarcinoma is the most frequently reported histologic subtype of jejunum neoplasm, and that not all cases of lymphoma debut with chylous ascites, it can be concluded that the proportion of patients that present with this condition is exceptionally low. Chyloperitoneum is an infrequent finding, having the higher detection rate in lymphatic alterations and malignancies of gastrointestinal location, in which some of the most commonly neoplasms associated with this complication are lymphoma, neuroendocrine tumors, sarcomas, and leukemia.
\end{abstract}

Keywords: chylous ascites; gastrointestinal neoplasms; case reports

\section{Introduction}

The lymph is a fluid that originates in the interstitial space composed of cells, proteins, chylomicrons, and occasionally bacteria. This fluid enters the lymphatic system, making its way to the lymph nodes which act as filters for the lymph, then entering the cisterna chyli, and later reaching the thoracic duct, following its path until it reaches the major circulation, draining at the level of the left subclavian and left internal jugular vein. A high percentage of the lymph originates in the abdominal organs (particularly the liver and intestine) [1]. When the structures of this system, more specifically its main ducts, are injured or obstructed, lymph spreads to the adjacent spaces causing a variety of conditions. If the above occurs in the context of a peritoneal effusion, it is referred to as chyloperitoneum or chylous ascites [2]. The diagnosis of chyloperitoneum is based on the clinical presentation of ascites through the analysis of the fluid, including a milky appearance and triglyceride levels $>200 \mathrm{mg} / \mathrm{dL}$ of the fluid [3]. Chyloperitoneum fluid 
is rich in biologically unavailable nutrients and immunoglobulins after accumulation in the peritoneum, which can lead to dehydration, malnutrition, electrolyte-hydric alteration, and immunosuppression [4].

The underlying etiologies of chyloperitoneum have been classified as congenital, traumatic, infectious, neoplastic, postoperative, cirrhotic, or cardiogenic [3]; almost twothirds of the reported cases in developed countries are of cirrhotic origin or associated with abdominal malignancy, while, in developing countries, infectious diseases such as tuberculosis account for the majority of cases [5].

Chyloperitoneum is considered an uncommon yet severe complication during abdominal surgery [6]. It occurs most often during postoperative retroperitoneal lymph node dissection, distal splenic shunt, abdominal aortic aneurysm repair, or liver transplant [5,7], while its presentation as a complication of colorectal cancer surgery is exceptional [8].

The incidence of chyloperitoneum has increased due to prolonged survival after surgical interventions in patients with cancer, as well as patients who have undergone aggressive cardiothoracic and abdominal interventions $[6,8]$. The prognosis and treatment of this disease varies depending on the underlying cause; considering that there is no series of studies where the sociodemographic and clinical data of those affected were thoroughly analyzed, its medium- or long-term impact are uncertain. Bearing the limited knowledge on this subject, especially in developing countries, it is proposed to present the case of a 56-year-old man who presented with chyloperitoneum as the initial manifestation of gastrointestinal lymphoma. Since epidemiologically speaking its presentation is secondary to a neoplasm in the described location, this is a key finding in the quest to understand and document the effects of chyloperitoneum. Informed consent was requested by the medical team for the publication of this case report and was signed by the patient, including images.

\section{Case}

A 56-year-old Black man, working as an accountant, from an urban area, with a clinical history of approximately three months of evolution. His symptoms consisted of the sensation of an abdominal mass, predominantly in the left hemiabdomen, associated with progressive abdominal distension, changes in bowel habits, lower limb edema, dyspepsia, occasional postprandial emesis, and unintended $20 \mathrm{~kg}$ weight loss. The patient presented no comorbidities or surgical history; in addition, arterial hypertension was the only concern in his family history. He was not taking any medication at the time of the evaluation. A total abdomen ultrasonography was performed. The report displayed a non-specific mesenteric mass at the root of the mesentery, abundant ascitic fluid, and a small liver with dilated portal vein. At the time of the assessment by the general surgery service, the patient was in an acceptable general condition with vital signs of: BP, 130/90 mmHg; HR, 92 beats/min; height, $160 \mathrm{~cm}$; weight, $52 \mathrm{~kg}$; and BMI, $20 \mathrm{~kg} / \mathrm{m}^{2}$. At the physical examination, globose abdomen was found, with a sensation of an irregular mass predominantly in the left hemiabdomen, positive ascitic wave, general pain, no signs of peritoneal irritation, and Grade III edema of the lower limbs. Within the initial paraclinical studies there was no evidence of alteration in the cell lines, the renal and hepatic function were preserved, and there were no electrolytic alterations.

Tumor marker studies were requested (CA 19.9, CEA, and AFP), all of which were negative. Endoscopic studies (esophagogastroduodenoscopy and colonoscopy) were performed with findings of gastropathy, but without evidence of lesions suggestive of neoplasia. A contrast abdominal tomography scan was performed where a retroperitoneal-looking mass was found measuring $154 \mathrm{~mm} \times 119 \mathrm{~mm}$ and extending to the mesogastrium, as well signs of ascites (Figures 1 and 2). A simple chest tomography revealed right pleural thickening associated with bilateral posterior-basal atelectasis without evidence of adjacent lesions. In addition, a diagnostic laparoscopy was scheduled to collect samples for the histopathological study. The findings during the procedure were: chyloperitoneum of $10,000 \mathrm{ccs}$ (Figure 3) and a large mass measuring $20 \mathrm{~cm} \times 20 \mathrm{~cm}$ involving the meso of the proximal jejunum, with fish meat appearance, and distension of the intestinal loops 
without evidence of obstruction. The chyloperitoneum was drained and lesion biopsies were taken.

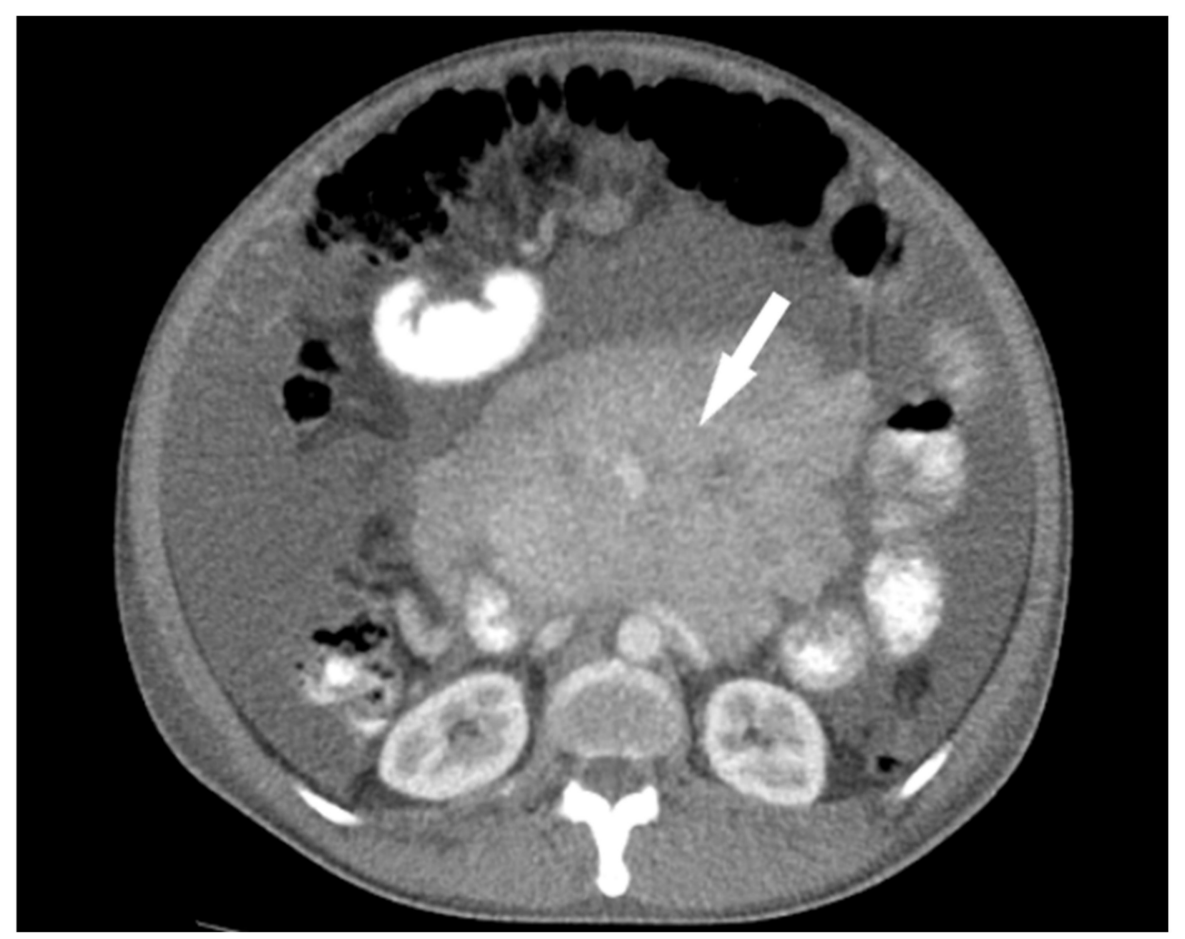

Figure 1. Tomographic image showing a retroperitoneal mass of $154 \mathrm{~mm} \times 119 \mathrm{~mm}$ that extends to the mesogastrium (white arrow).

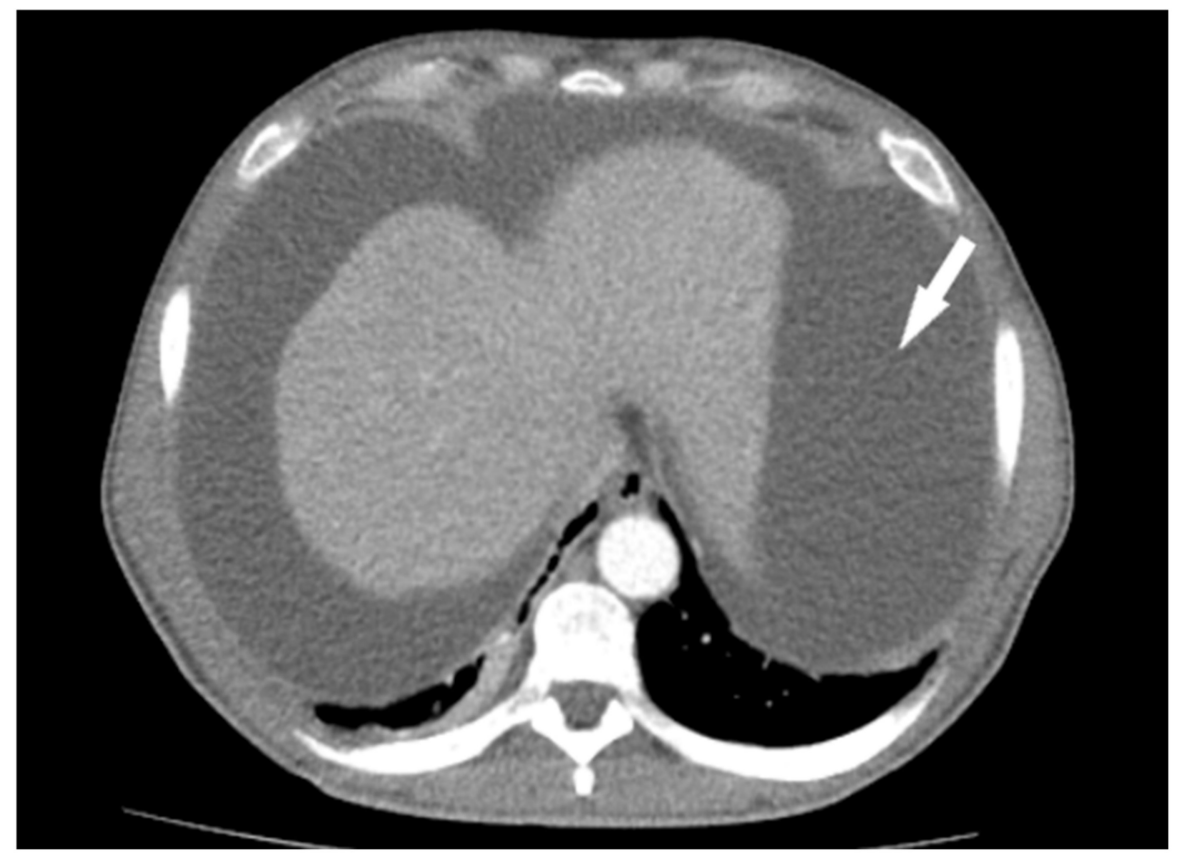

Figure 2. Tomographic image showing abundant fluid in the abdominal cavity: Signs of ascites (white arrow). 


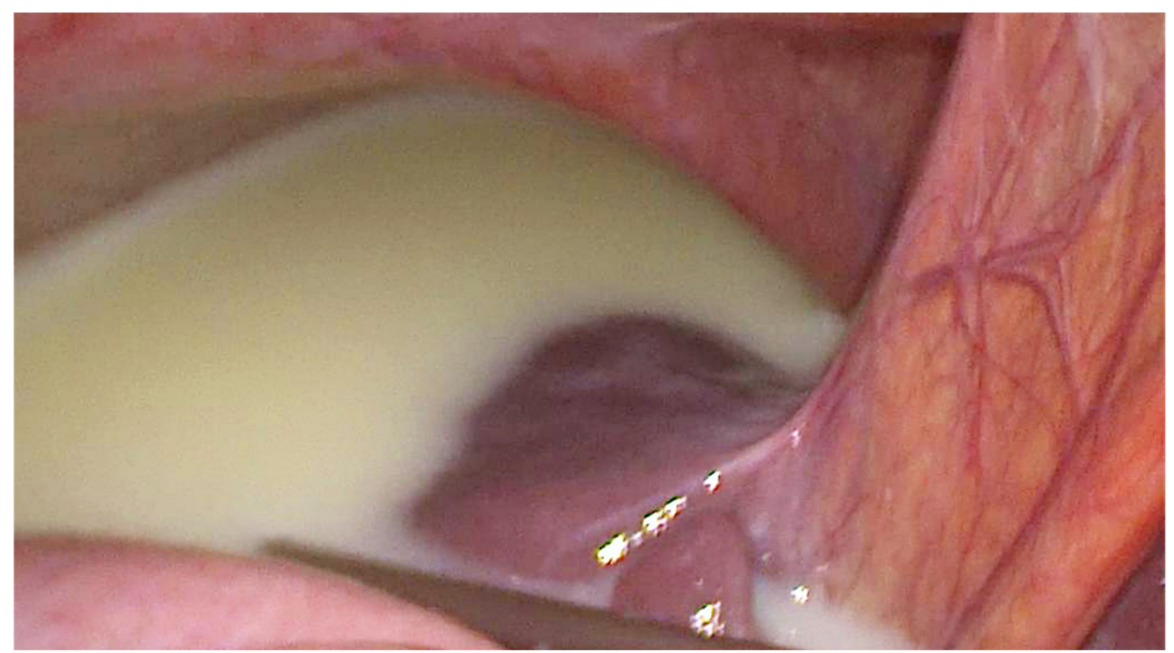

Figure 3. Intraoperative photography where chyloperitoneum is observed.

The patient evolved satisfactorily, tolerating diet on his first postoperative day, with adequate control of abdominal pain, improvement in intestinal transit, and marked decrease in ascites, without complications inherent to the surgical procedure. The patient was discharged on the fourth day after surgery. A follow up fifteen days after discharge demonstrated adequate recovery from the surgical intervention; no abdominal pain was reported. In addition, surgical wounds were healing properly, and no signs of ascites were found at the physical examination.

The peritoneal fluid cytological examination showed to contain large groups of lymphoid-like cells on a fibrinoid background, suggesting lymphoid neoplastic infiltration. Furthermore, anatomical pathology results show lymph node infiltration in diffuse form, small lymphocytes with hyperchromatic nuclei, and areas of sclerosis in the node's medulla. Consecutively, lymphoma was established as a definitive diagnosis.

\section{Discussion}

Pathophysiologically, chylous ascites can occur through three processes: acquired lymphatic disruption (dilation of retroperitoneal lymph vessels occurs, causing lymph extravasation to the abdomen through a fistula, which may be traumatic or iatrogenic), a congenital cause (such as congenital lymphangiectasia), or secondary to fibrosis of the lymphatic system (seen in malignancies, where there is lymph obstruction from the intestines to the cisterna chyli) [3,9]. One of the few systematic reviews of cases existing in the literature on chylous ascites mentions that the most frequent causes of nontraumatic chylous ascites in adults are malignancy $(25 \%)$, followed by cirrhosis $(16 \%)$ and mycobacterial infections (15\%) [10].

The most common symptoms described are abdominal distention and mild pain lasting weeks to months, with dyspnea, peritonitis, and weight gain in a few cases, due to increase in intra-abdominal pressure [10]. However, among all age groups, the lymphatic abnormalities are the most frequent cause (32\%), with lymphangiectasia being the most prevalent etiology and observed to a greater extent in the pediatric population (84\%) compared to the adult population (8\%) [10]. The second most frequent cause in all groups are the malignant entities, responsible for approximately $17 \%$ of cases of nontraumatic chylous ascites, with lymphoma, neuroendocrine tumors, sarcomas, and leukemia being the most frequently encountered causes [10]. The type of ascites secondary to malignant neoplasms is almost exclusively within the adult population, presenting only in $2 \%$ of cases in the infant population [6,11]. Taking into consideration that adenocarcinoma is the most frequently presented histologic subtype of cancer in the jejunum (58.7\%), followed by carcinoid tumor (16.5\%), lymphoma (9.7\%), and sarcoma (6.3\%) [11-13], and that not all cases of lymphoma debut with chylous ascites, it can be said that the percentage of patients 
that present this complication is very low. Considering its non-specific presentation, it is necessary to begin with a clinical history and detailed physical examination to discard the great possible number of possible differential diagnoses (Table 1).

Table 1. Differential diagnoses to consider in non-traumatic chylous ascites $[3-5,8,11]$.

\begin{tabular}{cc}
\hline Conditions & Cases (\%) \\
\hline Lymphatic abnormalities & $9-32$ \\
Malignant tumors in solid organs & $7-25$ \\
Sarcomas & $<9$ \\
Neuroendocrine tumors & 3 \\
Lymphomas & 5 \\
Cirrhosis & $11-16$ \\
Mycobacterial infections & $10-15$ \\
Pancreatitis & 4 \\
Congestive heart failure & 1 \\
Nephrotic syndrome & 3 \\
Vasculitis & 4 \\
Gastrointestinal disorders & 3 \\
Congenital diseases & 3 \\
Serositis & Undetermined \\
\hline
\end{tabular}

Abdominal paracentesis is the most important tool for the evaluation of chylous ascites since it allows identifying the distinctive characteristics of this fluid (milky, thick, and cloudy appearance). It is recommended to obtain a minimum sample of $100 \mathrm{~mL}$ [14]. Likewise, cell count, Gram staining, microbiological culture, evaluation of glucose levels, total proteins, albumin, amylase, lactate dehydrogenase, triglycerides, and cytology should be performed. In the case of suspected mycobacterial infection, resistant acid-alcohol staining and polymerase chain reaction are key [14]. For the definitive diagnosis of chylous ascites, electrophoresis must be performed for the detection of chylomicrons through lipoproteins; however, accessing this type of specialized studies is complex [15].

Cytology and peritoneal biopsy are important diagnostic methods if peritoneal carcinomatosis is suspected. It has shown a specificity of around $97 \%$ for cancer in the case of a positive cytology [16]. Similarly, biopsy through laparoscopy has been found to be overly sensitive and specific for the diagnosis of peritoneal cancer and tuberculosis. It should be noted that laparoscopy in these cases serves as both a diagnostic and therapeutic technique and highly useful in clinical pictures where the etiology is difficult to identify $[17,18]$.

Tomography has the function of identifying if there is an abdominal mass or affected lymph nodes that explain the picture presented. It also allows evaluating the amount of fluid in the peritoneal cavity, but it does not have the ability to distinguish between common ascitic fluid and chylous ascites because its imaging densities are similar [17].

There is no consensus on the management of patients with chylous ascites secondary to gastrointestinal neoplasia. The approach depends on the cause and the findings found, so the therapeutic process can range from a simple drainage process to chemotherapy and definitive surgery. A low-sodium diet should be established in order to reduce fluid retention and reduce edema. The use of diuretics is not necessary, since there are no reliable studies that support their use; they have only been effective in $45 \%$ of cases [18-20]. In addition, it has been found that somatostatin can control the production of chyloperitoneum by decreasing intestinal fat absorption, reducing the concentration of triglycerides in the thoracic duct, and regulating lymph flow in the major lymphatic vessels [21]. However, there is insufficient evidence to recommend this therapy.

\section{Conclusions}

Chyloperitoneum is an infrequent finding, being most often found in lymphatic alterations and malignancies of gastrointestinal location. Lymphoma, neuroendocrine tumors, sarcomas, and leukemia are the neoplasms most associated with this complication, 
occurring almost exclusively in the adult population. Depending on the local or regional epidemiology and patient history, a large number of possible differential diagnoses, ranging from traumatic causes to congenital diseases, can be discarded. The evaluation of the clinical picture should be complemented by physical examination, imaging studies, analysis of the ascitic fluid, and intraoperative biopsy. The treatment then depends on the cause and symptoms, being able to establish a non-invasive approach based on diet, pharmacological therapy, and peritoneal-venous shunts.

Author Contributions: Conceptualization, I.D.L.-M., P.Z.-R., V.H.M.-B. and A.M.R.-G.; methodology, I.D.L.-M., D.T.-L. and M.B.-R.; formal analysis, I.D.L.-M., P.Z.-R., V.H.M.-B. and A.M.R.-G.; investigation, I.D.L.-M., D.T.-L., P.Z.-R., V.H.M.-B., M.B.-R. and A.M.R.-G.; writing-original draft preparation, I.D.L.-M., D.T.-L., P.Z.-R., V.H.M.-B., M.B.-R. and A.M.R.-G.; writing-review and editing, I.D.L.-M., D.T.-L., P.Z.-R., V.H.M.-B., M.B.-R. and A.M.R.-G.; and supervision, I.D.L.-M., P.Z.-R., V.H.M.-B. and A.M.R.-G. All authors have read and agreed to the published version of the manuscript.

Funding: This research received no external funding.

Institutional Review Board Statement: The study was conducted according to the guidelines of the Declaration of Helsinki, and approved by ethics committee of Hospital Universitario San Jorge (14 August 2020).

Informed Consent Statement: Informed consent was obtained from all subjects involved in the study.

Data Availability Statement: Not applicable.

Conflicts of Interest: The authors declare no conflict of interest.

\section{References}

1. Kim, J.-H.; Han, E.-H.; Jin, Z.-W.; Lee, H.-K.; Fujimiya, M.; Murakami, G.; Cho, B.H. Fetal Topographical Anatomy of the Upper Abdominal Lymphatics: Its Specific Features in Comparison with Other Abdominopelvic Regions. Anat. Rec. Adv. Integr. Anat. Evol. Biol. 2011, 295, 91-104. [CrossRef] [PubMed]

2. Lopez-Gutierrez, J.C.; Tovar, J.A. Chylothoraz and chylous ascites. Management and pitfalls. Semin. Pediatr. Surg. 2014, 23, 298-302. [CrossRef] [PubMed]

3. Lizaola, B.; Bonder, A.; Trivedi, H.D.; Tapper, E.B.; Cardenas, A. Review article: The diagnostic approach and current management of chylous ascites. Aliment. Pharmacol. Ther. 2017, 46, 816-824. [CrossRef] [PubMed]

4. Dababneh, Y.; Mousa, O.Y. Chylous Ascites. In StatPearls [Internet]; StatPearls Publishing: Treasure Island, FL, USA, 2020.

5. Patil, A.; Nandikoor, S.; De Marco, J.; Bhat, R.; Shivakumar, S.; Mallrajapatna, G. Disorders of the lymphatic system of the abdomen. Clin. Radiol. 2016, 71, 941-952. [CrossRef] [PubMed]

6. Aalami, O.O.; Allen, D.B.; Organ, C.H. Chylous ascites: A collective review. Surgery 2000, 128, 761-778. [CrossRef] [PubMed]

7. Campisi, C.; Bellini, C.; Eretta, C.; Zilli, A.; Da Rin, E.; Davini, D.; Bonioli, E.; Boccardo, F. Diagnosis and management of primary chylous ascites. J. Vasc. Surg. 2006, 43, 1244-1248. [CrossRef] [PubMed]

8. Cardenas, A.; Chopra, S. Chylous ascites. Am. J. Gastroenterol. 2002, 97, 1896-1900. [CrossRef] [PubMed]

9. Al-Busafi, S.A.; Ghali, P.; Deschênes, M.; Wong, P. Chylous Ascites: Evaluation and Management. ISRN Hepatol. 2014, $2014,1-10$. [CrossRef] [PubMed]

10. Steinemann, D.C.; Dindo, D.; Clavien, P.-A.; Nocito, A. Atraumatic Chylous Ascites: Systematic Review on Symptoms and Causes. J. Am. Coll. Surg. 2011, 212, 899-905.e4. [CrossRef] [PubMed]

11. Almakdisi, T.; Massoud, S.; Makdisi, G. Lymphomas and Chylous Ascites: Review of the Literature. Oncologist 2005, 10, 632-635. [CrossRef] [PubMed]

12. Schottenfeld, D.; Beebe-Dimmer, J.L.; Vigneau, F.D. The Epidemiology and Pathogenesis of Neoplasia in the Small Intestine. Ann. Epidemiol. 2009, 19, 58-69. [CrossRef] [PubMed]

13. Bhardwaj, R.; Vaziri, H.; Gautam, A.; Ballesteros, E.; Karimeddini, D.; Wu, G.Y. Chylous Ascites: A Review of Pathogenesis, Diagnosis and Treatment. J. Clin. Transl. Hepatol. 2018, 6, 1-9. [CrossRef]

14. Thaler, M.A.; Bietenbeck, A.; Schulz, C.; Luppa, P.B. Establishment of triglyceride cut-off values to detect chylous ascites and pleural effusions. Clin. Biochem. 2017, 50, 134-138. [CrossRef]

15. Runyon, B.A.; Hoefs, J.C.; Morgan, T.R. Ascitic fluid analysis in malignancy-related ascites. Hepatology 1988, 8, 1104-1109. [CrossRef] [PubMed]

16. Chu, C.-M.; Lin, S.-M.; Peng, S.-M.; Wu, C.-S.; Liaw, Y.-F. The role of laparoscopy in the evaluation of ascites of unknown origin. Gastrointest. Endosc. 1994, 40, 285-289. [CrossRef]

17. Lee, C.W.; Bociek, G.; Faught, W. A Survey of Practice in Management of Malignant Ascites. J. Pain Symptom Manag. 1998, 16, 96-101. [CrossRef] 
18. Saif, M.W.; Siddiqui, I.A.; Sohail, M.A. Management of ascites due to gastrointestinal malignancy. Ann. Saudi Med. 2009, 29, 369-377. [CrossRef] [PubMed]

19. Murphy, M.; Rossi, M. Managing ascities via Tenckhoff catheter. Medsurg. Nurs. 1995, 4, 468-471. [PubMed]

20. Becker, G.; Galandi, D.; Blum, H.E. Malignant ascites: Systematic review and guideline for treatment. Eur. J. Cancer 2006, 42, 589-597. [CrossRef] [PubMed]

21. Karaca, S.; Gemayel, G.; Kalangos, A. Somatostatin treatment of a persistent chyloperitoneum following abdominal aortic surgery. J. Vasc. Surg. 2012, 56, 1409-1412. [CrossRef] [PubMed] 